\title{
Frequent clonal relations between metastases and non-index prostate cancer lesions
}

\author{
Jeroen Kneppers, ${ }^{1,2}$ Oscar Krijgsman, ${ }^{2,3}$ Monique Melis, ${ }^{4}$ Jeroen de Jong, ${ }^{2,5}$ Daniel S. Peeper, ${ }^{2,3}$ \\ Elise Bekers, ${ }^{5}$ Henk G. van der Poel, ${ }^{6}$ Wilbert Zwart, ${ }^{1,2,7}$ and Andries M. Bergman ${ }^{1,8}$ \\ 'Division of Oncogenomics, Netherlands Cancer Institute, Amsterdam, Netherlands. \\ ${ }^{2}$ Oncode Institute, Utrecht, Netherlands. ${ }^{3}$ Division of Molecular Oncology \& Immunology, \\ ${ }^{4}$ Division of Molecular Genetics, ${ }^{5}$ Division of Molecular Pathology, and ${ }^{6}$ Division of Urology, Netherlands Cancer \\ Institute, Amsterdam, Netherlands. 'Laboratory of Chemical Biology and Institute for Complex Molecular Systems, \\ Department of Biomedical Engineering, Eindhoven University of Technology, Eindhoven, Netherlands. \\ ${ }^{8}$ Division of Medical Oncology, Netherlands Cancer Institute, Amsterdam, Netherlands.
}

Primary prostate cancer lesions are clonally heterogeneous and often arise independently. In contrast, metastases were reported to share a monoclonal background. Because prostate cancer mortality is the consequence of distant metastases, prevention of metastatic outgrowth by primary tumor ablation is the main focus of treatment for localized disease. Focal therapy is targeted ablation of the primary index lesion, but it is unclear whether remaining primary lesions metastasize at a later stage. In this study, we compared copy number aberration profiles of primary prostate cancer lesions with matching pelvic lymph node metastases of 30 patients to establish clonality between a lymph node metastasis and multiple primary lesions within the same patient. Interestingly, in $\mathbf{2 3 . 3} \%$ of the cases, the regional metastasis was not clonally linked to the index primary lesion. These findings suggest that focal ablation of only the index lesion is potentially an undertreatment of a significant proportion of prostate cancer patients.

Conflict of interest: The authors have declared that no conflict of interest exists.

License: This work is licensed under the Creative Commons Attribution 4.0 International License.

Submitted: September 10, 2018 Accepted: December 11, 2018 Published: January 24, 2019

Reference information: JCI Insight. 2019;4(2):e124756 https://doi.org/10.1172/jici. insight.124756.

\section{Introduction}

Prostate cancer (PCa) has the highest incidence of all cancers in men worldwide (1). The majority of patients present with a tumor confined to the prostate and can be treated with curative intent. Prevention of metastatic outgrowth by local treatment of the prostate is a key objective of PCa treatment, as systemic metastatic spread is the main cause of mortality. For primary local treatment, a choice is made between radiotherapy (RT) and radical prostatectomy (RP), which are considered equally effective (2). However, both types of treatment are associated with significant adverse side effects, including urinary incontinence, impotence, and bladder and rectum dysfunction (3).

A more focal treatment of identified tumors in the prostate could spare men the toxicity of whole-gland treatments. However, PCa is often multifocal (4). In approximately $60 \%$ of patients, multiple subclones with distinct clinicopathological features are identified in the prostate at the time of diagnosis (5). Conventionally, the largest primary tumor with the highest grade is defined as the index lesion $(6,7)$. There is limited evidence that progression of the disease into disseminated PCa is linked to the index lesion $(8,9)$. Several approaches to focal therapy (FT) have been developed in recent years, aimed at ablating the index lesion and maintaining the oncological benefit of active treatments while preserving genitourinary and other organ functions (10).

However, a number of small reports indicated that metastatic disease does not uniformly originate from the index lesion but can also derive from small, obscure non-index primary lesions. Reports linking non-index lesions with metastases used fluorescence in situ hybridization analyses in 23 cases (11), targeted next-generation sequencing in a single patient case (12), and deep whole-genome sequencing in a cohort study of 10 cases (6). However, because most of these studies are statistically underpowered or used technologies that prevented a comprehensive interpretation of genomic data, it remains to be determined which percentage of lymph node (LN) metastases is clonally derived from a non-index lesion. 
Structural genomic rearrangements (SGRs) are common in PCa (13), and all SGRs cumulatively constitute a copy number alteration (CNA) profile. Profiling tumors for clonal SGRs reveals tumor evolutionary trajectories, and therefore CNA profiling can be applied to reveal clonality between primary lesions and metastases $(12,14,15)$.

In this study, we used low-coverage whole-genome sequencing (LC-WGS) to uncover CNA profiles of matched primary lesions and local metastases. In total, we have interrogated CNA profiles of 70 primary lesions and matched them with 30 local metastases from 30 patients. By comparing CNA profiles in patients, we aim to shed light on the primary clonal origins of local metastases of PCa. Interestingly, in $23.3 \%$ of cases, the index lesion was not the clonal origin of the local metastasis. This suggests that focal ablation of only the index lesion might be an undertreatment of a significant proportion of PCa patients.

\section{Results}

To investigate clonality between primary lesions and LN metastases, we obtained clinical data from a cohort of 30 patients who underwent RP and a pelvic LN dissection, with at least 1 primary tumor and $1 \mathrm{LN}$ metastasis identified (Table 1). We hypothesized that CNA profiles would enable us to assess clonality between metastases and primary lesions within the same patient. Multiple primary tumors are observed in the prostate gland (Figure $1 \mathrm{~A}$ ), of which the largest tumor with the highest grade is considered the index lesion $\left(\mathrm{P}_{1}\right)$. Other primary lesions $\left(\mathrm{P}_{2-\mathrm{n}}\right)$ can vary in size and grade, and pelvic LNs are a frequent site of regional PCa metastases.

Because genetically different primary lesions have unique CNA profiles, we here directly compared the CNA profiles of LN metastases with different primary lesions in the same patient to identify the disseminating primary lesion (Figure $1 \mathrm{~B}$ ). Our expert pathologist defined $\mathrm{P}_{1}$ based on tumor size and Gleason score and delineated the areas of the other primary lesions $\left(\mathrm{P}_{2-6}\right)$ in the prostate and site $\mathrm{N}$ in LNs (Figure 1C). $\mathrm{N}$ was selected based on the largest lesion to maximize the chance of successful DNA isolation. A total of 63 primary tumors were identified in 30 patients.

As an example, we discuss patient 11 (Supplemental Table 1; supplemental material available online with this article; https://doi.org/10.1172/jci.insight.124756DS1), from whom 3 primary tumors and a large LN metastasis are shown (Figure 1C) and whose primary tumors were also detected on MRI (Supplemental Figure 1). DNA was isolated from all visible primary tumors $\mathrm{P}_{1-6}$ and $\mathrm{N}$. Additionally, as a control, we isolated DNA from 2 extra sites of index lesion $\mathrm{P}_{1}$ (denoted $\mathrm{P}_{1}^{*}$ and $\mathrm{P}_{1}^{* *}$ in figures) and performed LC-WGS for CNA profiles of all DNA samples. CNA profiles of $\mathrm{P}_{1}$ and $\mathrm{N}$ shared common CNA profile features, while $\mathrm{P}_{2}$ and $\mathrm{N}$ did not, suggesting that $\mathrm{N}$ disseminated from $\mathrm{P}_{1}$ (Figure 1D). Pearson correlation (Figure 1E) and a principal component analysis (Figure 1F) both showed a correlation between $\mathrm{P}_{1}$ and $\mathrm{N}$ versus $\mathrm{P}_{2-6}$

To estimate the percentage of patients with regional metastases that did not disseminate from the primary PCa index lesion, we selected 30 FFPE prostatectomy specimens and concurrent regional metastases (Table 1). Among all 30 patients, previously reported frequent CNAs $(16,17)$ were identified, including deletions at TMPRSS2-ERG, PTEN, and chromosome 8p (Figure 2A). Globally, no major differences were observed in aggregate CNAs between primary lesions and regional metastases (Figure 2, B and C). We did not find clear copy number enrichment of specific regions when comparing primary tumors that metastasized versus those that did not. However, given the relatively limited resolution of the data, focal gains and losses are likely overlooked, and thus, conclusive statements on focal aberrations cannot be made at this stage.

Both a $\log \mathrm{LR}$ and $P$ value of clonality were calculated for pairs of $\mathrm{P}-\mathrm{N}$ samples compared to a reference distribution representing the average profile of all samples (18). All intrapatient pairs were divided in 2 groups to separate index lesions ( $\mathrm{P}_{1}-\mathrm{N}$ pairs) from other primary lesions $\left(\mathrm{P}_{2-\mathrm{n}}-\mathrm{N}\right.$ pairs $)$. We defined pairs to be clonal for LR $P$ values of less than 0.01 and observed more clonal $\mathrm{P}_{1}-\mathrm{N}$ pairs than $\mathrm{P}_{2-\mathrm{n}}-\mathrm{N}$ pairs (Figure 3A). To assess the percentage of clonality for index lesions and LN metastases, we compared the similarity of breakpoints of CNA heatmaps for individual patients (Figure 3B). Interestingly, in 23 out of 30 patients (76.7\%), $\mathrm{P}_{1}$ was clonal with $\mathrm{N}$ (Figure $3 \mathrm{~B}$ ), and consequently in $23.3 \%$ of patients, the metastasis was not clonally related to the index lesion. For most cases, the other smaller foci studied were not clonally linked with the metastasis either, and only for 1 case we managed to connect a non-index lesion with the metastasis analyzed (Supplemental Figure 2). Based on these findings, we conclude that in most of these patients, the metastasis originated from an undetected, obscure primary lesion, as was reported earlier (12). 


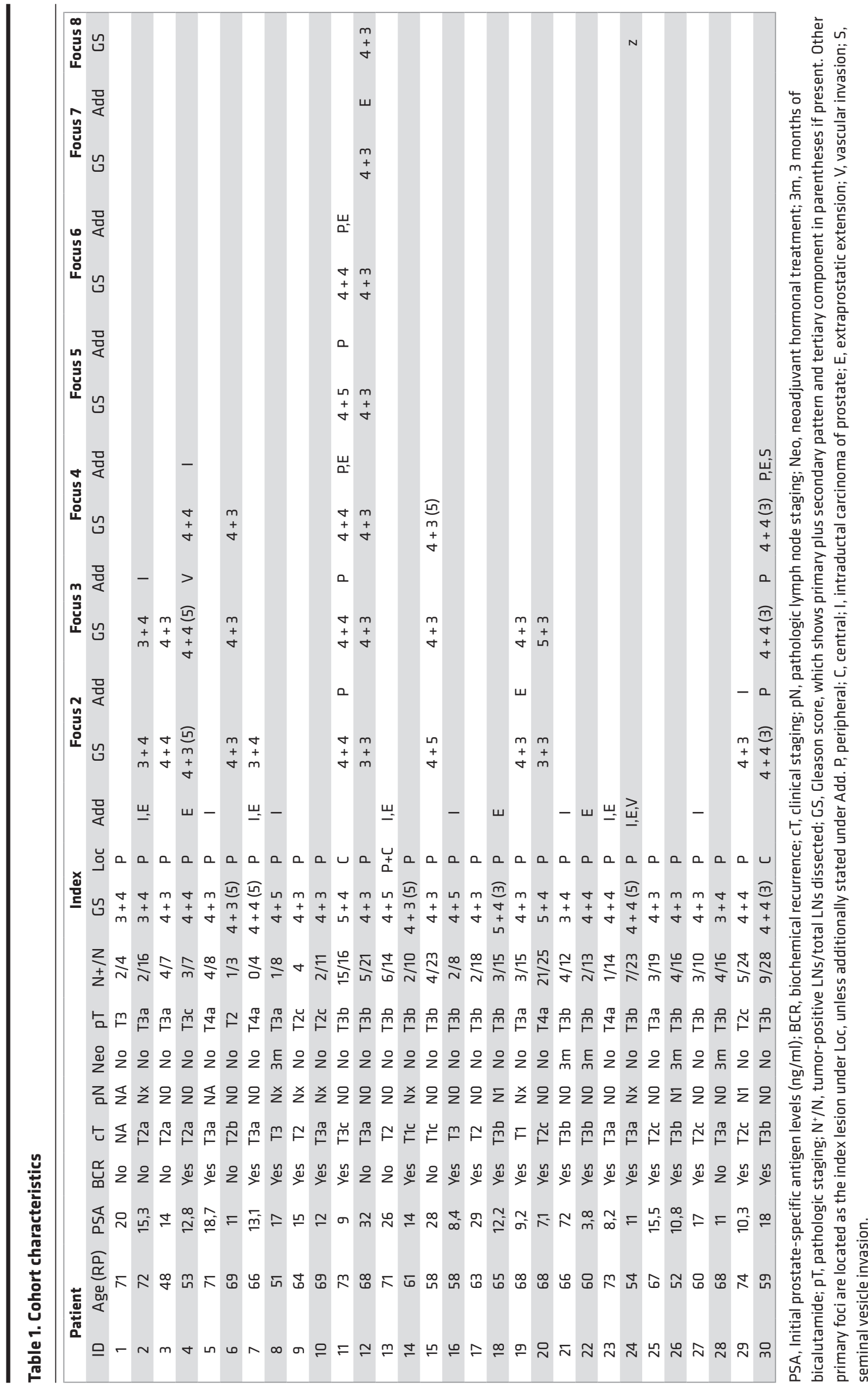


A

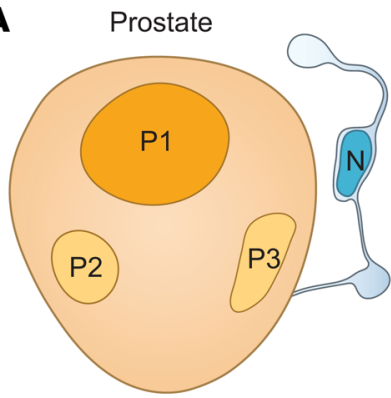

B

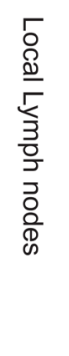

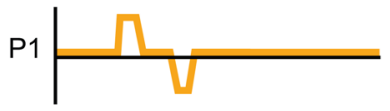

P2

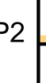

$\mathrm{N}$

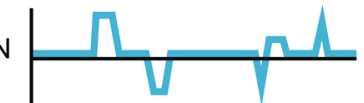

C

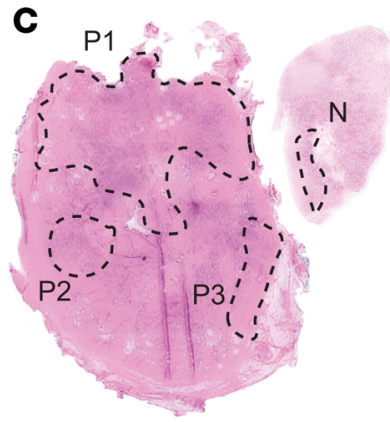

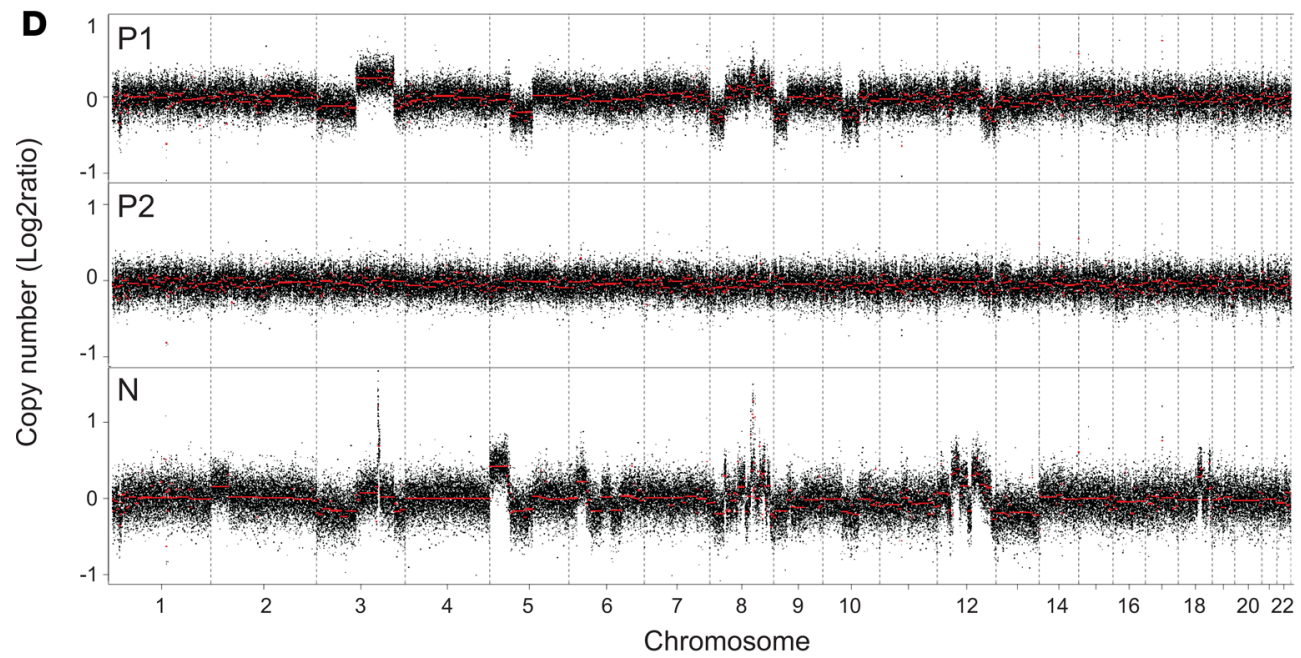

E

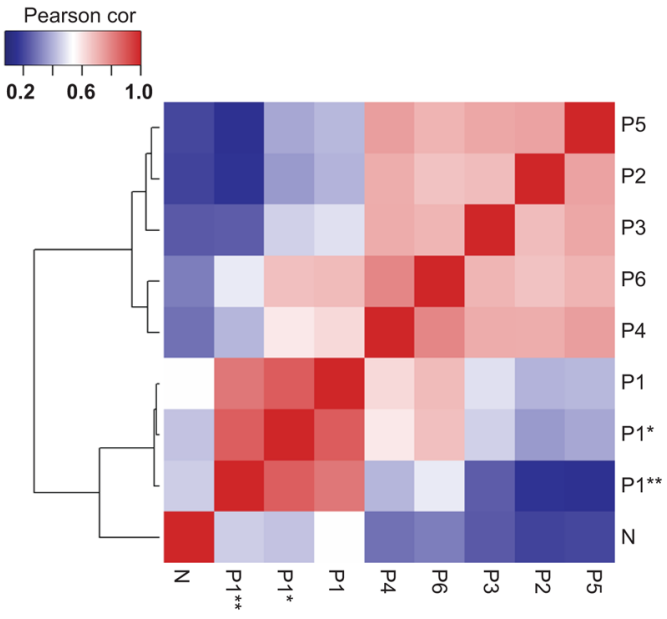

$\mathbf{F}$

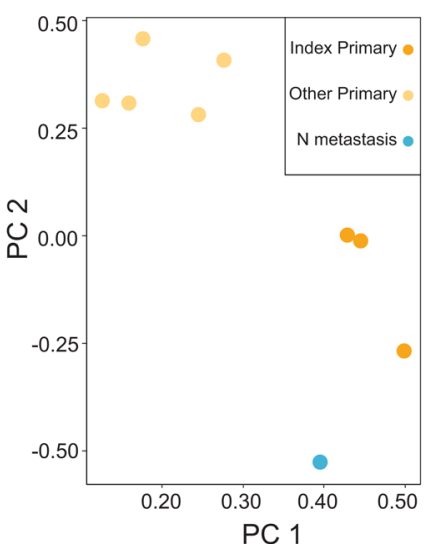

Figure 1. Case study of patient 11. (A) Schematic representation of a prostate with multiple primary lesions $(P)$ and a pelvic LN metastasis (N). (B) Example CNA profiles showing different SGR features in the index lesion $\mathrm{P}_{1}$, other primary lesion $P_{2}$, and $L N$ metastasis $N$. Breakpoint features are shared between $N$ and $P_{1}$. (C) H\&E staining of the FFPE whole-mount prostatectomy and an LN metastasis of patient 11. The index lesion $P_{1}$ and 2 other primary lesions $\left(P_{2}\right.$ and $\left.P_{3}\right)$ as well as the LN metastasis are indicated. Marks in $P_{1}$ where 3 cores were taken are indicated. (D) Selected CNA profiles of patient 11 acquired by LC-WGS and processed with QDNAseq (see Methods). Black dots represent log2 ratio values in bins of $30 \mathrm{~kb}$; red lines represent segmented log2 ratio values. (E) Pearson correlation heatmap of segmented CGHcall (15) values of all isolated tumors of patient 11. (F) Principal component analysis plot of segmented CGHcall values of $\mathrm{P}_{1}, \mathrm{P}_{2-\mathrm{n}}$, and $\mathrm{N}$. 
A
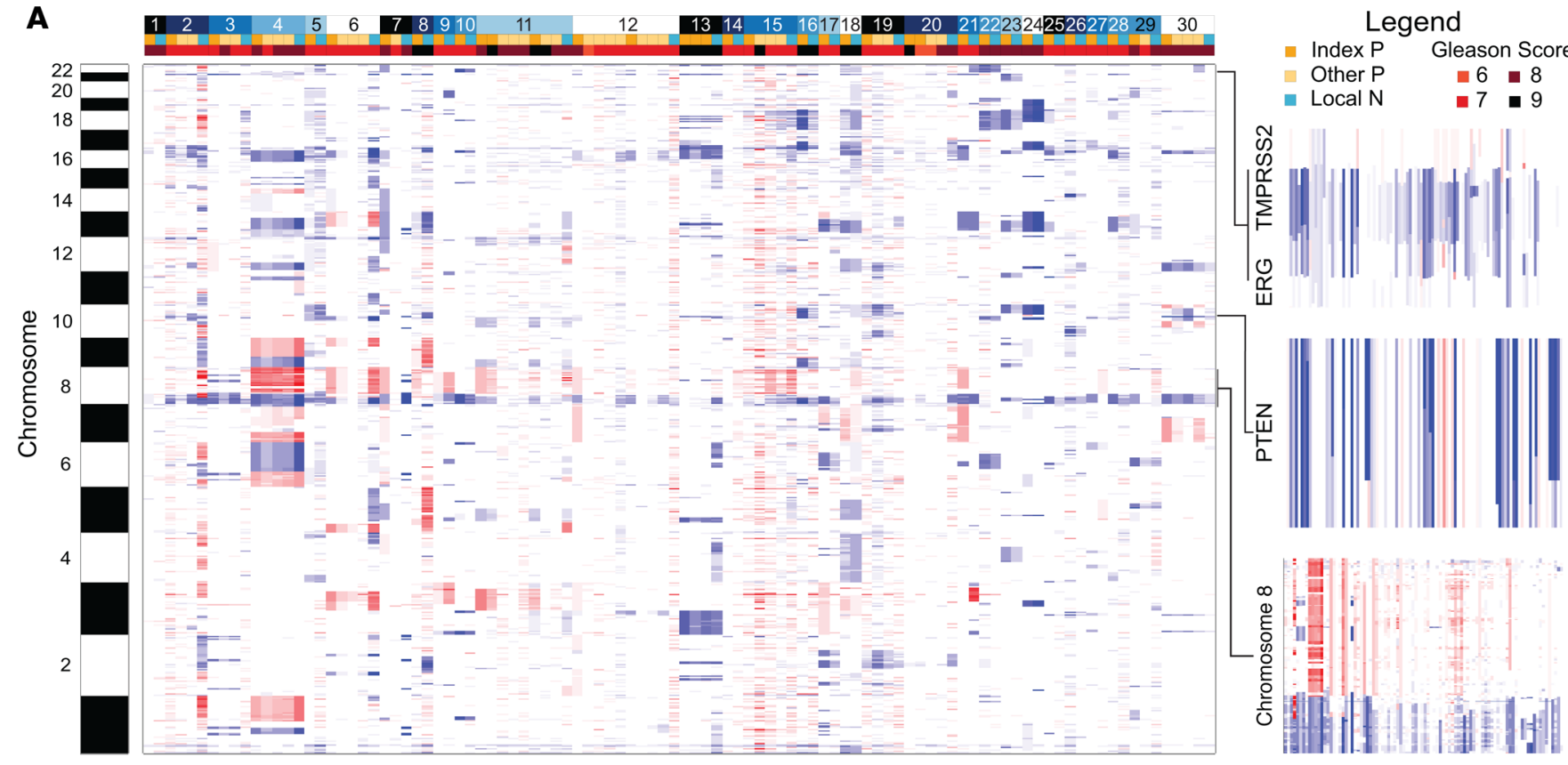

Local N $\quad 7=9$

๗ู
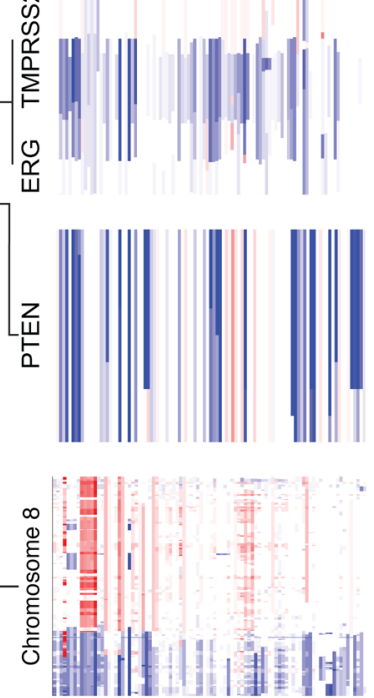

B

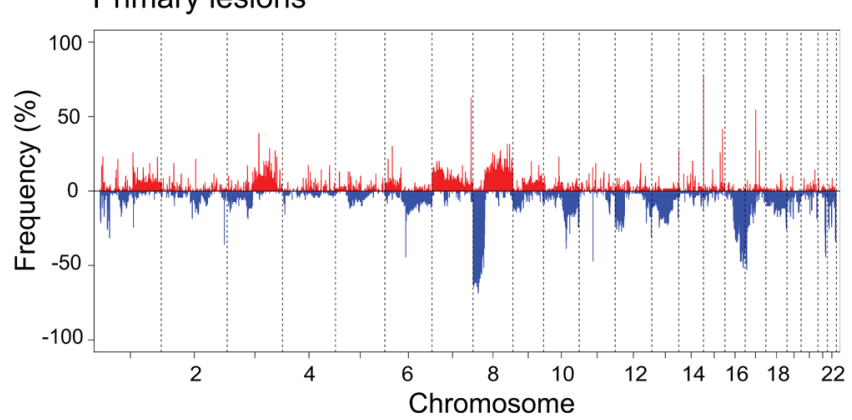

C Local metastases

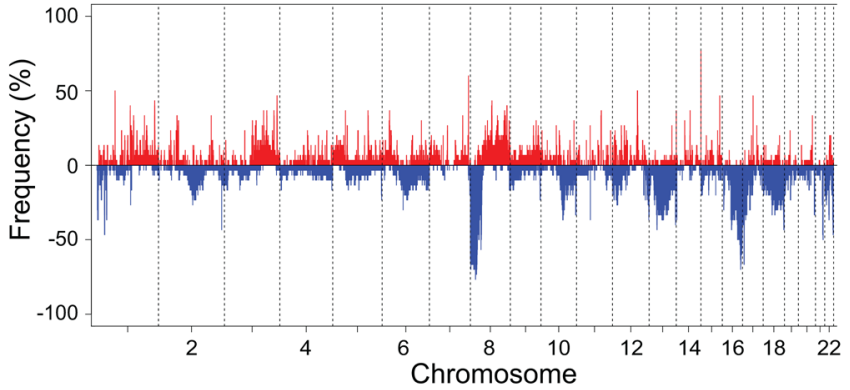

Figure 2. CNA profiles of PCa lesions and LN metastases in entire cohort. (A) CNA overview heatmap of 30 patients with zoomed-in regions of interest, showing typical PCa SCRs, such as chromosome 21 TMPRSS2-ERG focal deletions, chromosome 10 PTEN deletions, and chromosome $8 \mathrm{q}$ arm loss and 8p gain. Known blacklisted genomic loci were excluded, showing amplifications (red) and deletions (blue). (B) Aggregate frequency plot of all primary lesion CNAs, $n=70$. (C) Aggregate frequency plot of all LN metastasis CNAs, $n=30$.

\section{Discussion}

In this study, we identified a clonal connection between primary tumors and a single LN metastasis, which could be considered a limited view on metastatic disease progression. The original paradigm of linear progressive metastatic seeding is increasingly giving way to an emerging view of more diverse patterns of subclonal metastatic seeding, such as (a)synchronous polyclonal seeding and cross-metastatic seeding (19, 20). LN metastases are not necessarily an intermediate step for the development of distant metastases but can also represent a genetic dead end in tumor phylogenies (21).

Robust comparative data on medium- to long-term oncological outcomes of FT versus whole-prostate gland treatments are currently lacking (10). Although FT is considered experimental, it is offered to patients with low to intermediate $(<50 \%)$ risk of PCa recurrence (19). Implementation of FT without any risk of recurrence would require extensive preoperative biopsies (4), which would be impractical and can still easily miss small lesions.

However, important questions on the relation between primary lesions and lethal disease remain. Which primary lesion contributed to PCa recurrence after targeted ablation when any primary lesion theoretically has the potential to metastasize? Because we did not sample distant metastases, future studies should be aimed to yield more comprehensive insights into metastatic spread. Although we identified multiple tumor-positive LNs in some patients, successful FFPE DNA extraction requires both high tumor 
A

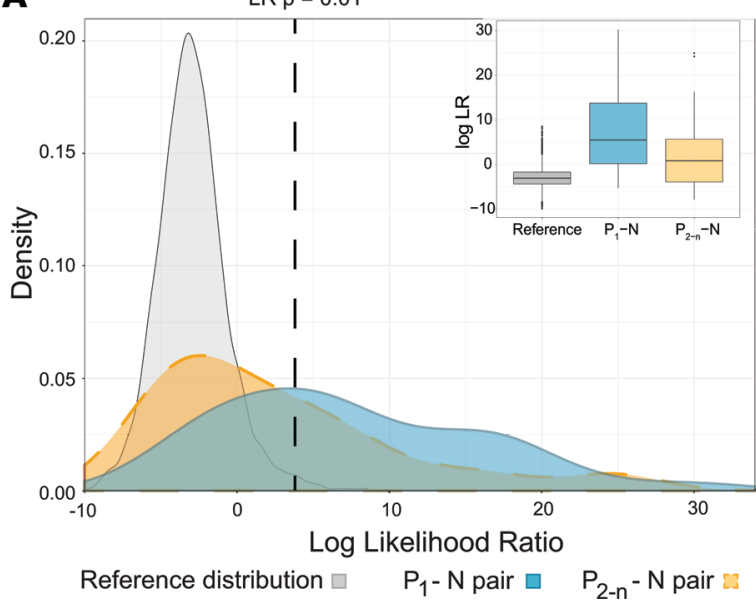

B

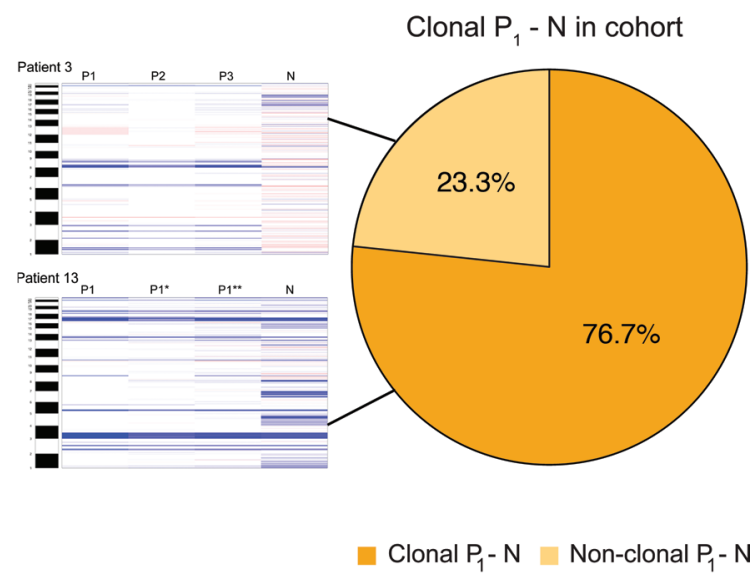

Figure 3. Clonality assessment of PCa lesions and LN metastases. (A) Density plot of log likelihood ratio (LR) of 3 pair groups: reference distribution, $\mathrm{P}_{1}-\mathrm{N}$ pairs, and $\mathrm{P}_{2-n}-\mathrm{N}$ pairs. A box plot representation of the density plot is shown in the corner. Dotted line represents $L R P$ value of 0.01 , which is considered significant. (B) Intrapatient CNA heatmap comparison of $\mathrm{P}_{1}$ and $\mathrm{N}$, indicating that $23.3 \%$ of $L N$ metastases are not clonally related to the primary index lesion. Two CNA heatmaps from 2 patients are shown as examples. Patient 3 shows no clonality between $\mathrm{P}_{1}$ and $\mathrm{N}$. Patient 13 shows clonality between $\mathrm{P}_{1}$ and $\mathrm{N}$ based on identical breakpoints in genomes.

cellularity and sufficient size of the LN metastasis being analyzed. Due to this restriction, we focused our analysis on clonality between primary tumors and the biggest tumor-positive LN. However, PCa metastases were reported to share a monoclonal background (7), suggesting that assessing a single metastasis captures the early evolutionary history of metastatic PCa.

Interestingly, we found that in $23.3 \%$ of cases the index lesion was not the clonal origin of the $\mathrm{LN}$ metastasis in a high-risk cohort of patients who underwent a prostatectomy and a pelvic lymphadenectomy. Despite the difference in risk classification between patients offered FT and our cohort, it is highly likely that in a significant proportion of patients treated with FT, the primary source of metastatic lesions remains in situ. This suggests that a significant proportion of FT-treated patients are potentially undertreated and would consequently have a higher risk of PCa recurrence. Accordingly, our results urge for randomized trials comparing oncological outcomes of patients treated with RP or RT as compared to FT.

\section{Methods}

Patient selection. We selected 30 patients from the Netherlands Cancer Institute Antoni van Leeuwenhoek (NKI-AVL) biobank based on availability of a local LN metastasis size greater than $4 \mathrm{~mm}$. Clinicopathological characteristics of this cohort are shown in Table 1.

Sample acquisition and DNA isolation. All tissues were processed into FFPE material by the molecular pathology division of the NKI-AVL using standard protocols. For determining tumor areas, 5- $\mu \mathrm{m}$ sections of FFPE prostate and local metastasis samples were prepared. H\&E staining was performed according to standard protocols. Our expert pathologist delineated areas with a tumor cell percentage higher than $60 \%$ for all visible primary lesions and local metastases. Sample acquisition was performed by collecting 0.6 $\mathrm{mm}$ cores from the FFPE block in tumor areas. Core biopsies of primary tumors, metastases, and normal prostate tissue of all samples in the cohort were included in a tissue microarray (TMA). TMAs of the RP tissue were constructed using a manual tissue arrayer (Beecher Instruments). TMAs of the LN metastasis were constructed using the automated TMA Grand Master (3DHISTECH). Depending on availability, 2 or 3 different cassettes per tumor were used for TMA construction, and 3 cores of $0.6-\mathrm{mm}$ diameter were put in the TMA. H\&E slides were cut to assess the quality of the TMA.

An additional dropout TMA was constructed for tumor cores that had low core quality. Finally, DNA was isolated from TMA FFPE cores using standard protocols. High-throughput sequencing data were deposited in the NCBI's Sequence Read Archive (SRP178079).

Sequencing. Isolated DNAs were preprocessed and obtained DNA libraries were analyzed on a 2100 Bioanalyzer using a 7500 chip (Agilent), diluted, and pooled equimolar into a 10-plex sequencing pool (Illumina Inc.). The libraries were sequenced with 65 base single reads on a HiSeq 2500 system using 
version 4 chemistry (Illumina Inc). Obtained reads were mapped to Homo sapiens reference genome hg19 using BWA-backtrack (23). In general, we achieved an average genome coverage of $0.2-1$ times.

QDNAseq. Mapped sequence reads obtained with LC-WGS were analyzed using QDNAseq by R (24) with a 30-kb window and default settings. The median normalized $\log 2$ ratios from QDNAseq were segmented with circular binary segmentation (25). After segmentation, samples were mode normalized and chromosomal copy number changes defined using CGHcall by R (15).

Pearson correlation heatmap, principal component analysis, frequency plots, and CNA heatmaps. The Pearson correlation matrix was calculated based on the segmented $\log 2$ ratio values for patient 11 and plotted with heatmap.3 (26) using ward.D2 hierarchical clustering. Next, we performed a principal component analysis on segmented $\log 2$ ratio values to reduce dimensionality to 2 dimensions.

CGHcall data were plotted in both a genome-wide and zoomed-in CNA profile heatmap for the entire cohort. Moreover, we plotted aggregate CNA frequencies for 2 selected groups: primary tumors and metastases from combined CGHcall data.

Clonality $R$ package. For each set of DNA copy number profiles from a single patient, we calculated the likelihood 2 samples were clonal. This was performed using the Clonality $\mathrm{R}$ package with default settings. CGHcall CNA data were downsampled 40-fold for optimal performance of the package as Ostrovnaya et al. described (18).

Statistics. Clonality package log LR ratio statistics were computed as described originally (18). Onetailed Student's $t$ test was used to compare the means among reference, $\mathrm{P}_{1}-\mathrm{N}$, and $\mathrm{P}_{2-\mathrm{n}}-\mathrm{N}$ groups.

Study approval. This study was performed in accordance with institutional medical ethical guidelines. The use of anonymous or coded leftover material for scientific purposes is part of the standard treatment agreement with patients, and therefore informed consent was not required from patients not actively opting out, according to Dutch law (22). The NKI-AVL has set up a secondary-use biobank under the conditions in "Human Tissue and Medical Research: Code of Conduct for Responsible Use" (Federa, 2011, http://www.federa.org/codes-conduct). The use of NKI-AVL biobank material requires review and approval by NKI-AVL's Translational Research Board (TRB) according to the aforementioned Code of Conduct. Patient material was used only after approval by the TRB when otherwise disposed of or unnecessary for diagnostics.

\section{Author contributions}

WZ and AMB conceived the study. JK, WZ, and AMB conceived experiments. JK carried out experiments. MM, HGVDP, EB, JDJ, and AMB provided tissues, samples, and clinical information. JK analyzed data with the help of OK. The manuscript was written by JK, WZ, and AMB with input from all the other authors.

\section{Acknowledgments}

The authors express their gratitude to the staff at the NKI Genomics Core Facility for sequencing support and the staff at the NKI Core Facility Molecular Pathology \& Biobanking for their technical and logistical support. WZ is supported by a research grant from the KWF Dutch Cancer Society (10084 ALPE), a KWF Dutch Cancer Society/Alpe d'HuZes Bas Mulder award (NKI 2014-6711), and a VIDI grant (016.156.401) from The Netherlands Organisation for Scientific Research. AMB is supported by a research grant from the KWF Dutch Cancer Society (KWF grant 2009-4356).

Address correspondence to: Wilbert Zwart or Andries M. Bergman, Plesmanlaan 121, Amsterdam 1066CX, Netherlands. Phone: 31.20.512.2101; Email: w.zwart@nki.nl (WZ). Phone: 31.20.512.2569; Email: a.bergman@nki.nl(AMB).

1. Zhou CK, et al. Prostate cancer incidence in 43 populations worldwide: An analysis of time trends overall and by age group. Int J Cancer. 2016;138(6):1388-1400.

2. Mottet N, et al. EAU-ESTRO-SIOG Guidelines on Prostate Cancer. Part 1: Screening, Diagnosis, and Local Treatment with Curative Intent. Eur Urol. 2017;71(4):618-629.

3. Chade DC, et al. Cancer control and functional outcomes of salvage radical prostatectomy for radiation-recurrent prostate cancer: a systematic review of the literature. Eur Urol. 2012;61(5):961-971. 
4. Løvf M, et al. Multifocal primary prostate cancer exhibits high degree of genomic heterogeneity [published online ahead of print September 1, 2018]. Eur Urol. doi: 10.1016/j.eururo.2018.08.009.

5. Espiritu SMG, et al. The evolutionary landscape of localized prostate cancers drives clinical aggression. Cell. 2018;173(4):1003-1013.e15

6. Gundem G, et al. The evolutionary history of lethal metastatic prostate cancer. Nature. 2015;520(7547):353-357.

7. Liu W, et al. Copy number analysis indicates monoclonal origin of lethal metastatic prostate cancer. Nat Med. 2009;15(5):559-565.

8. Wise AM, Stamey TA, McNeal JE, Clayton JL. Morphologic and clinical significance of multifocal prostate cancers in radical prostatectomy specimens. Urology. 2002;60(2):264-269.

9. Algaba F, Montironi R. Impact of prostate cancer multifocality on its biology and treatment. J Endourol. 2010;24(5):799-804.

10. Valerio M, et al. New and established technology in focal ablation of the prostate: a systematic review. Eur Urol. 2017;71(1):17-34.

11. Gburek BM, Kollmorgen TA, Qian J, D'Souza-Gburek SM, Lieber MM, Jenkins RB. Chromosomal anomalies in stage D1 prostate adenocarcinoma primary tumors and lymph node metastases detected by fluorescence in situ hybridization. $J$ Urol. 1997;157(1):223-227.

12. Haffner MC, et al. Tracking the clonal origin of lethal prostate cancer. J Clin Invest. 2013;123(11):4918-4922.

13. Barbieri CE, Rubin MA. Genomic rearrangements in prostate cancer. Curr Opin Urol. 2015;25(1):71-76.

14. McGranahan N, Swanton C. Clonal heterogeneity and tumor evolution: past, present, and the future. Cell. 2017;168(4):613-628.

15. van de Wiel MA, Kim KI, Vosse SJ, van Wieringen WN, Wilting SM, Ylstra B. CGHcall: calling aberrations for array CGH tumor profiles. Bioinformatics. 2007;23(7):892-894.

16. Camacho N, et al. Appraising the relevance of DNA copy number loss and gain in prostate cancer using whole genome DNA sequence data. PLoS Genet. 2017;13(9):e1007001.

17. Armenia J, et al. The long tail of oncogenic drivers in prostate cancer. Nat Genet. 2018;50(5):645-651.

18. Ostrovnaya I, Seshan VE, Olshen AB, Begg CB. Clonality: an R package for testing clonal relatedness of two tumors from the same patient based on their genomic profiles. Bioinformatics. 2011;27(12):1698-1699.

19. Hong MK, et al. Tracking the origins and drivers of subclonal metastatic expansion in prostate cancer. Nat Commun. $2015 ; 6: 6605$.

20. Macintyre G, Van Loo P, Corcoran NM, Wedge DC, Markowetz F, Hovens CM. How subclonal modeling is changing the metastatic paradigm. Clin Cancer Res. 2017;23(3):630-635.

21. Mangiola S, et al. Comparing nodal versus bony metastatic spread using tumour phylogenies. Sci Rep. 2016;6:33918.

22. van Diest PJ. No consent should be needed for using leftover body material for scientific purposes. For. $B M J$. 2002;325(7365):648-651.

23. Li H, Durbin R. Fast and accurate short read alignment with Burrows-Wheeler Transform. Bioinformatics. 2009;25(14):1754-1760.

24. Scheinin I, et al. DNA copy number analysis of fresh and formalin-fixed specimens by shallow whole-genome sequencing with identification and exclusion of problematic regions in the genome assembly. Genome Res. 2014;24(12):2022-2032.

25. Olshen AB, Venkatraman ES, Lucito R, Wigler M. Circular binary segmentation for the analysis of array-based DNA copy number data. Biostatistics. 2004;5(4):557-572

26. Zhao S. heatmap3 v1.1.1. R documentation website. https://www.rdocumentation.org/packages/heatmap3/versions/1.1.1 Accessed December 17, 2018. 